\title{
EMBEDDINGS IN $G(1,3)$
}

\section{A. PAPANTONOPOULOU}

\begin{abstract}
We give a description of nonsingular surfaces of degree $d \leqslant 8$ in the Grassmannian $G(1,3)$ embedded in $\mathbf{P}^{5}$.
\end{abstract}

1. Preliminaries. Let $G=G(1,3)$ be the Grassmann variety of projective lines in a fixed $\mathbf{P}^{3}$. Fix an embedding of $G$ in $\mathbf{P}^{5}$. Let $Y \subset G$ be a nonsingular surface. This embedding is uniquely determined by a short exact sequence of vector bundles on $Y$,

$$
0 \rightarrow E \rightarrow O_{Y}^{4} \rightarrow Q \rightarrow 0,
$$

where rank $Q=\operatorname{rank} E=2$ and $\wedge^{2} Q$ is the very ample line bundle embedding $Y$ in $\mathbf{P}^{5}$. In the Chow ring $A(G)$ of $G, Y \sim a Z_{1}+b Z_{2}$, where the $Z_{i}$ are the two Schubert cycles of dimension 2 in $A(G), a=c_{2}(Q), b=c_{2}(\check{E})$, and the degree of $Y$ in $\mathbf{P}^{5}$ is $d=c_{1}^{2}(Q)=c_{1}^{2}(\check{E})=a+b$. If $Y \neq Z_{i}$, then $a, b>0$. We denote by $(a, b)$ the class of $Y$ in $A(G)$. For background see [9]. Throughout we work over $\mathbf{C}$.

Proposition 1.1. With $Y$ and $(a, b)$ as above, let $H$ be a hyperplane section on $Y, K$ the canonical divisor on $Y$, and $N_{Y / G}$ the normal bundle on $Y$ in $G$. Then

$$
a^{2}+b^{2}=c_{2}\left(N_{Y / G}\right)=7 d+4 K H+2 K^{2}-12-12 p_{a}(Y) \text {. }
$$

Proof. By [9, Appendix] the tangent bundle of $G$ restricted on $Y$ is $T_{G Y}=Q \otimes \check{E}$. Hence, tensoring (1) by $\check{E}$ and calculating total Chern classes, we get

$$
c_{1}\left(T_{G Y}\right)=4 c_{1}(\check{E})=4 H \text { and } c_{2}\left(T_{G Y}\right)=7 c_{1}^{2}(\check{E})=7 d .
$$

Hence, from the short exact sequence $0 \rightarrow T_{Y} \rightarrow T_{G Y} \rightarrow N_{Y / G} \rightarrow 0$, we get

$$
c_{1}\left(N_{Y / G}\right)=4 H+K \text { and } c_{2}\left(N_{Y / G}\right)=7 d-c_{2}\left(T_{Y}\right)+4 K H+K^{2} \text {. }
$$

(2) follows by Riemann-Roch. Q.E.D.

Proposition 1.2. With $Y$ and $(a, b)$ as above, let $C$ be a nonsingular curve on $Y$ representing the hyperplane section $H$. Assume $Y$ lies in $\mathbf{P}^{4}$. Then:

(i) The genus of $C$ is $g=a b-(a+b)+1$.

(ii) If $d=2 n$, then $(a, b)=(n, n)$ and $Y$ is a complete intersection, $Y=G \cap \mathbf{P}^{4} \cap$ $S_{n}$, where $S_{n}$ is a hypersurface in $\mathbf{P}^{5}$ of degree $n$.

Proof. $Y \subset \mathbf{P}^{4}$ and, hence, from [5, p. 434] we have

$$
d^{2}-10 d-5 H K-2 K^{2}+12+12 p_{a}(Y)=0 .
$$

Received by the editors June 10, 1982 and, in revised form, April 12, 1983.

1980 Mathematics Subject Classification. Primary 14M15; Secondary 14J99, 14 E25. 
From (2) we have $d^{2}-\left(a^{2}+b^{2}\right)-3 d-H K=0$, and (i) follows from the adjunction formula.

For (ii) we can assume $Y$ does not lie in $\mathbf{P}^{3}$. (Indeed, if $Y \subset \mathbf{P}^{3}$, then $Y$ is a nonsingular quadric, $a=b=1$, and $Y=G \cap \mathbf{P}^{3}$.) $Y \subset Z=G \cap \mathbf{P}^{4}$. If $Z$ is nonsingular, then $\operatorname{Pic}(Z) \cong \operatorname{Pic}(G) \cong \mathbf{Z}$, and $Y$ is a complete intersection $Z \cap S_{n} \cap \mathbf{P}^{4}$. Hence, $C$ is a complete intersection and (ii) follows from [6, Theorem 1]. If $Z$ is singular, then since $d=2 n$, (ii) follows by [5, p. 352]. Q.E.D.

For a nonsingular surface $Y$ in $G$ ruled over a nonsingular curve $T$, we fix the following notation: $\pi: Y \rightarrow T$ is the natural projection, $\varphi: Y \rightarrow Y_{0}$ is a birational morphism where $Y_{0}$ is geometrically ruled over $T, \varphi(C)=X=m T_{0}+n f$ in $Y_{0}$, where $C$ is a nonsingular curve representing a hyperplane section on $Y, e=$ $-\left(T_{0} \cdot T_{0}\right)_{Y_{0}}$.

$Y_{0}$ is obtained from $Y$ by blowing down $r$ exceptional divisors, and $r_{p}$ is the multiplicity of a point $p \in X$ where $p=\varphi(E)$ where $E$ is an exceptional divisor in $Y$. We have

$$
\begin{gathered}
X^{2}=m(2 n-m e)=C^{2}+\sum_{p} r_{p}^{2}, \\
2 g(X)-2=X^{2}+m e-2 n-m\left(2 p_{a}\left(Y_{0}\right)+2\right), \\
g(X)=g(C)+\frac{1}{2} \sum_{p} r_{p}\left(r_{p}-1\right), \quad K_{Y_{0}}^{2}=K_{Y}^{2}+r .
\end{gathered}
$$

In the next section we will also use the following

Proposition 1.3 [4, Propositions 3.1, 3.2]. Let $Y$ be a rational ruled surface and $C$ a nonsingular curve on $Y$. Then there exists a birational morphism $\varphi: Y \rightarrow F_{e}$, for some $e$, with the following properties:

(a) every multiple point of $\varphi(C)=X$ has multiplicity $r_{p} \leqslant m / 2$;

(b) if $e=0$, then $m \leqslant n$,

(c) if $e=1$, then $r_{p} \leqslant n$;

(d) if $m \geqslant 4$ and either $e \neq 1$, or $e=1$ and $n \geqslant m / 2$, then $C^{2} \leqslant \frac{2 m}{m-2}(g(C)-1)$,

(e) if $m \geqslant 4, e=1$ and $n<m / 2$, then

$$
C^{2}<\frac{2 k}{k-3}(g(C)-1), \text { where } k=n+m \text {. }
$$

Proposition 1.4 [1, p. 115]. Let $Y$ be a surface in $\mathbf{P}^{n}$ not contained in a hyperplane. Let $d=\operatorname{deg}(Y)$. Then:

(i) If $Y$ is not ruled, then $d \geqslant 2 n-2$, with equality holding only if $K=0$.

(ii) If $Y$ is ruled, then $0 \geqslant p_{a}(Y) \geqslant n-d-1$.

2. Surfaces of degree $d \leqslant 8$.

LEMMA 2.1. Let $H$ be a very ample divisor on a nonsingular curve $C$ that embeds $C$ in $\mathbf{P}^{n}$ so that $C$ does not lie in $\mathbf{P}^{n-1}$. Let $d$ be the degree of $C$. If $d \geqslant 2 g+1$, then $g=d-n$.

Proof. $H$ is a nonspecial divisor on $C$. Hence $l(H)=d-g+1$. By [7, p. 55] the embedding of $C$ by $H$ is projectively normal. Thus $l(H)=N+1$. Q.E.D. 
LEMmA 2.2. Let $Y$ be a nonsingular surface in $\mathbf{P}^{n}$ that does not lie in a $\mathbf{P}^{n-1}$. Assume $\operatorname{deg}(Y) \geqslant 2 g_{C}+1$, where $g_{C}$ is the genus of the nonsingular curve $C$ given by $a$ hyperplane section. Then $H^{1}\left(O_{Y}(m)\right)=0$ for all $m$.

Proof. $C \subset \mathbf{P}^{n-1}$ is projectively normal. Hence, from the commutative diagram

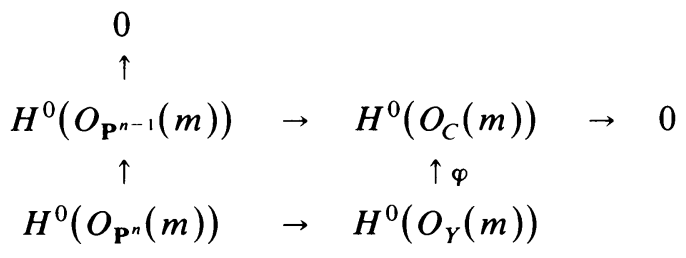

we get that $\varphi$ is surjective for all $m$. Hence $0 \rightarrow H^{1}\left(O_{Y}(m-1)\right) \rightarrow H^{1}\left(O_{Y}(m)\right)$ is injective for all $m$. Serre's Vanishing Theorem implies $H^{1}\left(O_{Y}(m)\right)=0$ for all $m$. Q.E.D.

THEOREM 2.3. Let $Y$ be a nonsingular surface in $G \subset \mathbf{P}^{5}$ of degree $d \leqslant 8$ not the projection of a surface in $\mathbf{P}^{6}$, and let $(a, b)$ be its class in the Chow ring $A(G)$ of $G$. Then one of the following holds:

(i) $d=1,(a, b)=(1,0), Y=\mathbf{P}^{2}$;

(ii) $d=2,(a, b)=(1,1), Y=F_{0}$;

(iii) $d=3,(a, b)=(2,1), Y=F_{1}$,

(iv) $d=4$, and either $(a, b)=(2,2)$ and $Y=F_{0}, F_{2}$, or the del Pezzo $S_{4}$, or else $(a, b)=(1,3)$ and $Y=$ the Veronese surface;

(v) $d=5,(a, b)=(2,3)$, and either $Y=F_{e}$ with 3 points blown up, or $Y=F_{e}$ with 7 points blown up;

(vi) $d=6,(a, b)=(3,3)$ and either $Y=F_{e}$ with 6 points blown up, or $Y=G \cap$ $\mathbf{P}^{4} \cap S_{3}$ and is a $K 3$ surface;

(vii) $d=7, Y=F_{e}$ with 8 points blown up, or $Y$ is the cubic surface with 5 points blown up, or $K^{2}=-12+6 p_{a}$;

(viii) $d=8,(a, b)=(4,4)$, and either $Y$ is the complete intersection of three quadrics, $Y=F_{0}$ with 10 points blown up, or $Y=G \cap \mathbf{P}^{4} \cap S_{4}$ is a surface of general type.

Proof. (i) and (ii) are obvious.

(iii) If $d=3$, then $Y \subset \mathbf{P}^{4}, g(C)=0, H K=-5$, and by Proposition $1.4, p_{a}=0$. By (2), $K^{2}=8$ and $Y$ is geometrically ruled. From (4) we get $e=1, m=1, n=2$.

(iv) If $d=4$ and $Y \not \subset \mathbf{P}^{4}$, then by Lemma $2.1, g(C)=0, H K=-6$, and $p_{a}=0$. If $(a, b)=(1,3)$, then by $(2), K^{2}=9$. If $(a, b)=(2,2)$, then $K^{2}=8$. If $Y \subset \mathbf{P}^{4}$, then by Proposition 1.2(ii), $Y$ is a complete intersection of two quadrics in $\mathbf{P}^{4}$, hence a del Pezzo $S_{4}$, with $(a, b)=(2,2)$.

(v) If $d=5$ and $Y \not \subset \mathbf{P}^{4}$, then $g(C)=1$ and $K H=-5$. By Proposition 1.4, $Y$ is ruled and by Lemma $2.2, p_{a}=0$. Hence from (2), $K^{2}=7$ or 5 . From Proposition $1.3, m \leqslant 3$. Hence by (4), $X^{2}=5+r=8, K^{2}=5$, and $(a, b)=(2,3), m=2$, $e=n-2$. 
But if $Y \subset \mathbf{P}^{4}, g(C)=2, K H=-3, Y$ is ruled, and hence by Lemma 2.2, $p_{a}=0$. From Proposition 1.3 we have $m \leqslant 3$. If $m=2$, then $e=n-3$, and if $m=3$, then $3 e=2(n-2)$. In either case, from (2) and (4) we get $r=7$ and $(a, b)=(2,3)$.

(vi) If $d=6$ and $Y \not \subset \mathbf{P}^{4}$, then $g(C)=2$ and $Y$ is ruled, hence from Lemma 2.2, $p_{a}=0$. By Proposition 1.3, $m \leqslant 3$. If $m=2$, then $e=n-3$. If $m=3$, then $3 e=2(n-2)$. In either case from (2) and (4) we get $r=6$.

But if $Y \subset \mathbf{P}^{4}$, by Proposition 1.2(ii), $Y$ is a complete intersection, $\omega_{y}=O_{Y}, Y$ is a $K 3$ surface, and $(a, b)=(3,3)$.

(vii) If $d=7$ and $Y \not \subset \mathbf{P}^{4}$, then $g(C)=3, K H=-3$, and $Y$ is a ruled surface with $p_{a}=0$ by Lemma 2.2. Hence by (2), $K^{2}=6$ or 2 or 0 . From Proposition $1.3, m \leqslant 4$. From (4) one can easily show that $m \neq 2$. If $m=3$, then $X^{2}=15$ and $K^{2}=0$ (and $3 e=2 n-5$ ). If $m=4$, let $r=s+t$, where $s$ is the number of points in $X$ with $r_{p}=1$, and $t$ is the number of points in $X$ with $r_{p}=2$. Then $X^{2}=7+s+4 t=$ $4(2 n-4 e)$, and $g(X)=3+t$. Hence from (4) we get $27=3 s+4 t$, and $s=5$, $t=3$. Hence $2 e=n-3, K^{2}=0$, and $(a, b)=(3,4)$.

But if $Y \subset \mathbf{P}^{4}$, then $g(C)=6$ or 4. If $g(C)=4$, then by [10 and 3, Theorem 3.1], $p_{a}=0$, hence $K^{2}=-2$, and $Y$ is the cubic surface with 5 points blown up. If $g(C)=6$, then $K^{2}=-12+6 p_{a}$.

(viii) If $d=8$ and $Y \not \subset \mathbf{P}^{4}$, then $g(C) \geqslant 4$. If $g(C)=4$, then by [3, Theorem 3.1], $p_{a}=0$ and $K^{2}=-2$, hence $(a, b)=(4,4)$ and $Y$ is the blowing up of a nonsingular quadric surface at 10 points. If $g(C) \geqslant 5$, then the very ample divisor $H$ on $C$ is special. Moreover $\operatorname{dim}|H|=\operatorname{deg}(H) / 2$. Hence by [5, p. 343], $H=K_{C}$, the canonical divisor on $C, C$ is not hyperelliptic, and $C$ is a complete intersection. Therefore $Y$ is a complete intersection of three quadrics in $\mathbf{P}^{5}, \omega_{Y}=O_{v}$, and by $(2),(a, b)=(4,4)$.

But if $Y \subset \mathbf{P}^{4}$ then by Proposition 1.2, $Y$ is a complete intersection $Y=G \cap \mathbf{P}^{4}$ $\cap S_{4}, K=H, p_{a}=5, Y$ is a surface of general type, and $(a, b)=(4,4)$. Q.E.D.

\section{REFERENCES}

1. A. Beauville, Surfaces algébriques complexes, Astérisque 54 (1978).

2. E. Bomberi and D. Husemoller, Classification and embeddings of surfaces, Proc. Symp. Pure Math., vol. 29, Amer. Math. Soc., Providence, R.I., 1975, pp. 329-420.

3. L. Ein, Nondegenerate surfaces of degree $n+3$ in $P_{C}^{n}$, preprint.

4. R. Hartshorne, Curves with high self-intersection on algebraic surfaces, Inst. Hautes Études Sci. Publ. Math. 36 (1969), 111-125.

Heidelberg, 1977

5. Algebraic geometry, Graduate Texts in Math., no. 52, Springer-Verlag, New York and

6. K. Kulek, The normal bundle of a curve on a quadric, Math. Ann. 258 (1981), 201-206.

7. D. Mumford, Abelian varieties, Oxford Univ. Press, New York and London, 1970.

8. M. Nagata, On rational surfaces. I, Mem. Colloq. Sci. Kyoto Ser. A 32 (1960), 351-370.

9. A. Papantonopoulou, Curves in Grassmann varieties, Nagoya Math. J. 66 (1977), 121-137.

10. L. Roth, On surfaces of sectional genus four, Proc. Cambridge Philos. Soc. 29 (1933), 184-194.

Department of Mathematics, Lehigh University, Bethlehem, Pennsylvania 18015 